\title{
-NOTES-
}

\section{ON ANALOGOUS THEOREMS OF FREDHOLM AND FRAME AND ON THE INVERSE OF A MATRIX*}

\section{By DANIEL SHANKS (Naval Ordnance Laboratory)}

In this note we give a simple proof of Frame's method of obtaining the characteristic equation of a matrix, and, by giving Fredholm's method of solving his integral equation a simple non-determinantal formulation, we show that the Frame and Fredholm algorithms are identical. On the basis of this identity we investigate the possibility of approximating the inverse of a matrix by means of an abbreviated computation.

Let $A$ be an arbitrary $N \times N$ matrix and let

$$
\lambda^{N}+p_{1} \lambda^{N-1}+p_{2} \lambda^{N-2}+\cdots+p_{n}=0
$$

be its characteristic equation. Let 0 and $I$ be the null and unit $N \times N$ matrices and define recursively the matrices, $A_{n}$, and the numbers, $a_{n}$, by:

$$
\begin{gathered}
A_{0}=0, \quad a_{0}=1, \\
A_{n}=A\left(A_{n-1}+a_{n-1} I\right), \quad a_{n}=-\frac{1}{n}\left(\operatorname{trace} A_{n}\right) . \quad(n=1,2, \cdots) .
\end{gathered}
$$

Then Frame shows (in [1], but in a different notation) that

$$
a_{n}=p_{n}
$$

and

$$
A_{N+1}=0 .
$$

A simpler proof may be adapted from the earlier work of Hotelling, Bingham and Girshick by using the theorem of Newton on the powers of the roots of a polynomial [2]. If $\lambda_{1}, \lambda_{2}, \cdots \lambda_{N}$ are the roots of (1), and if

$$
\begin{gathered}
s_{n}=\sum_{i=1}^{N} \lambda_{i}^{n}, \\
p_{1}=-s_{1}, \quad p_{2}=-\frac{1}{2}\left(s_{2}+p_{1} s_{1}\right)
\end{gathered}
$$

and, in general, $n=1,2, \cdots, N$,

$$
p_{n}=-\frac{1}{n}\left(s_{n}+p_{1} s_{n-1}+\cdots+p_{n-1} s_{1}\right) .
$$

Now for the matrix $A$,

$$
s_{n}=\operatorname{trace} A^{n}
$$

and since, from (2),

$$
A_{n}=A^{n}+a_{1} A^{n-1}+\cdots+a_{n-1} A,
$$

*Received February 8, 1954. 
by computing the trace of both sides we see from (6) that Eq. (3) follows by induction. Further

$$
A_{N}+a_{N} I=A^{N}+a_{1} A^{N-1}+\cdots+a_{N-1} A+a_{N} I=0
$$

by the Cayley-Hamilton theorem. Then Eq. (4) is immediate.

Since Frame shows that

$$
A_{N}+a_{N} I=A\left(A_{N-1}+a_{N-1} I\right)+a_{N} I=0,
$$

the inverse of $A$ is given by

$$
A^{-1}=-\left(A_{N-1}+a_{N-1} I\right) / a_{N}
$$

if it exists. We now note that it also follows that if $B=I-A$ then

$$
B^{-1}=I+\frac{\sum_{0}^{N} A_{n}}{\sum_{0}^{N} a_{n}}
$$

if it exists.

Alternatively we may develop (8) as a corollary of the Fredholm theory of integral equations. This approach yields useful information concerning the rate of convergence of the two series in (8) and the possibility of approximating (8) by replacing the $N$ there by some smaller integer, $r$. Fredholm's solution of

$$
u(x)=f(x)+\lambda \int_{a}^{b} K(x, y) u(y) d y
$$

may be written without determinants in a simple formulation which is virtually identical with (2) above. For from [3], Eqs. (15), (17) and (21) it may be seen that if we write

$$
\begin{gathered}
K_{0}(x, y)=0, \quad k_{0}=1, \\
K_{n}(x, y)=\int_{a}^{b} K(x, t) K_{n-1}(t, y) d t+k_{n-1} K(x, y), \quad(n=1,2, \cdots) \\
k_{n}=-\frac{1}{n} \int_{a}^{b} K_{n}(t, t) d t,
\end{gathered}
$$

then the resolvent,

$$
R(x, y)=\frac{\sum_{0}^{\infty} K_{n}(x, y) \lambda^{n}}{\sum_{0}^{\infty} k_{n} \lambda^{n}},
$$

is a meromorphic function of $\lambda$ such that Fredholm's solution of (9) is

$$
u(x)=f(x)+\int_{a}^{b} R(x, y, \lambda) f(y) d y .
$$

(It is perhaps worth noting in passing that if we replace $k_{n}=-(1 / n) \int_{a}^{b} K_{n}(t, t) d t$ in (10) by

$$
k_{n}=0 \quad(n=1,2, \cdots)
$$

we obtain, instead, the "iterated" kernels $K^{(n)}(x, y)$ and the Neumann series.)

Now let $a=0, b=N$, and assume that $K(x, y)$ is a two-dimensional $N \times N$ step 
function and that $f(x)$ is a one-dimensional $N$ step function. That is for

$$
\begin{aligned}
& i-1 \leqq x<i \\
& j-1 \leqq y<j
\end{aligned} \quad(i, j=1,2, \cdots, N)
$$

we assume

and

$$
\lambda K(x, y)=a_{i i} \quad \text { (a constant) }
$$

$$
f(x)=f_{i} \quad \text { (a constant). }
$$

Then for $A=\left(a_{i j}\right)$ we find by comparison of (2) and (10) that $K_{n}(x, y) \lambda^{n}=A_{n}$ and $k_{n} \lambda^{n}=a_{n}$. Thus from (11) and (12), we see that for this degenerate kernel, $K(x, y)$, Fredholm's solution of (9) may be expressed matrix-wise:

$$
u=\left[I+\frac{\sum_{0}^{N} A_{n}}{\sum_{0}^{N} a_{n}}\right] f .
$$

However for this degenerate kernel we may also solve the linear system (9) directly:

$$
u=(I-A)^{-1} f,
$$

and a comparison of the two solutions gives us (8) again.

But this time we get more, for in proving that $R(x, y, \lambda)$ is a meromorphic function of $\lambda$, that is, in proving the uniform convergence for all $\lambda$, of the two series in (11), Fredholm shows that if

$$
|K(x, y)| \leqq M^{*}
$$

then

$$
\left|K_{n}(x, y)\right| \leqq \frac{n^{n / 2}}{(n-1) !} \cdot M^{* n}(b-a)^{n-1} .
$$

In matrix notation we thus find that if

$$
\left|a_{i i}\right| \leqq \lambda M^{*}=M,
$$

then the elements of $A_{r}$ are bounded in magnitude by $b\left(A_{r}\right)$, where

$$
b\left(A_{r}\right)=r^{r / 2} M^{r} N^{r-1} /(r-1) !
$$

Similarly

$$
\left|a_{r}\right| \leqq r^{r / 2} M^{r} N^{r} / r !
$$

Therefore

$$
\left|\sum_{r+1}^{N} a_{n}\right|<\sum_{r+1}^{\infty} n^{n / 2}(M N)^{n} / n !<\frac{(r+1)^{(r+1) / 2}(M N)^{r+1}}{(r+1) !} \sum_{i=0}^{\infty}\left(\frac{M N e^{1 / 2}}{(r+1)^{1 / 2}}\right)^{i} .
$$

If we use Stirling's formula

$$
\frac{1}{n !}<\frac{1}{(2 \pi n)^{1 / 2}}\left(\frac{e}{n}\right)^{n}
$$


and let $z=e M N(r+1)^{-\frac{1}{2}}$ this becomes

$$
\left|\sum_{r+1}^{N} a_{n}\right|<\frac{1}{[2 \pi(r+1)]^{1 / 2}} \frac{z^{r+1}}{1-z e^{-1 / 2}} .
$$

Therefore if $M$ is sufficiently small, that is, if $z \ll 1$ for some $r<N$, it becomes feasible to approximate (8) by

$$
B^{-1} \approx I+\frac{\sum_{0}^{r} A_{n}}{\sum_{0}^{r} a_{n}} .
$$

If we do so we are assured that the error in the denominator will be no larger than (16) and similarly the errors in the elements of the numerator will be no greater than

$$
\frac{(r+1)^{1 / 2}}{N(2 \pi)^{1 / 2}} \cdot \frac{z^{r+1}}{1-z[(r+1) / e r]^{1 / 2}} .
$$

\section{REFERENCES}

1. J. S. Frame, $A$ simple recursion formula for inverting a matrix, Abs. 471, Bull. AMS 65, 1045 (1949). See also, Henry E. Fettis, Quart. Appl. Math. 8, 207 (1950).

2. Paul S. Dwyer, Linear computations, Wiley, 1951, pp. 223. See also, Chrystal, Textbook of algebra, Chelsea, 1952, Vol. I, pp. 437.

3. Lovitt, Linear integral equations, Dover, 1950, Chap. III.

\section{THE ELASTIC SPHERE UNDER CONCENTRATED TORQUES ${ }^{1}$}

\section{BY ALFRED HUBER (University of Maryland)}

1. Introduction. In this note we consider an elastic sphere subject to two concentrated torques acting at the end points of a diameter. The stress function occurring in this problem may be considered as a generalized axially symmetric potential ${ }^{2}$. This viewpoint enables us to obtain the solution by a simple potential-theoretic argument.

The notation used in this article is the same as that used by Timoshenko in [6].

2. Stress function. We introduce cylindrical coordinates $(r, \theta, z)$ and consider the elastic sphere $z^{2}+r^{2} \leqq 1$, subject to unit torques acting at the points $r=0, z= \pm 1$. All relevant quantities appear in a meridian $(z, r)$-plane. According to the theory of torsion of circular shafts we must find a stress function $\Phi(z, r)$ satisfying the following conditions: $\Phi$ is to be a solution of

$$
\frac{\partial^{2} \Phi}{\partial z^{2}}+\frac{\partial^{2} \Phi}{\partial r^{2}}-\frac{3}{r} \frac{\partial \Phi}{\partial r}=0
$$

defined and bounded in the half-circular region $H=\left[r^{2}+z^{2}<1\right] \cap[r>0]$, assuming the boundary value 0 on the open diameter, $1 / 2 \pi$ on the open circular boundary. This problem shall in the sequel be referred to as problem $(\mathrm{P})$.

${ }^{1}$ Received May 1, 1954. This research was supported by the United States Air Force through the Office of Scientific Research of the Air Research and Development Command.

${ }^{2}$ For the definition of this concept the reader is referred to [8]. 